\title{
FORMAL GROUPS AND THEIR ROLE IN THE APPARATUS OF ALGEBRAIC TOPOLOGY
}

\author{
V. M. BUKHSHTABER, A. S. MISHCHENKO, AND S. P. NOVIKOV \\ Dedicated to Ivan Georgievich Petrovskii on his seventieth birthday
}

\section{INTRODUCTION}

The present short survey forms a natural companion to that of Novikov [13], and it is helpful to read them together. Here we shall be mainly concerned with results in the development of cobordism theory in the last five years, based on the work of the authors, of Quillen and others. As an application we describe the beautiful work of Sullivan on the so-called Adams conjecture in $K$-theory.

\section{$\S 1$. FORMAL GROUPS}

In the modern topological apparatus constructed from cobordism theory, the theory of commutative formal groups and their generalizations plays an important part. Here we give the necessary introduction to this theory.

Let $A$ he a commutative associative ring with identity, $A\left[x_{1}, \ldots, x_{n}\right]$ the ring of polynomials in $x_{1}, \ldots, x_{n}$ with coefficients in $A$ and $A\left[\left[x_{1}, \ldots, x_{n}\right]\right]$ the corresponding ring of formal power series.

Definition 1.1. A commutative one-dimensional formal group over $A$ is a power series $F(u, v) \in A[[u, v]]$ such that $F(F(u, v), w)=F(u, F(v, w))$ and $F(u, v)=$ $F(v, u)$, where $F(u, 0)=u$.

Note that the existence of an "inverse element" $\varphi(u) \in A[[u]]$ such that $F(u, \varphi(u))=$ 0 follows from Definition 1.1.

Definition 1.2. A homomorphism $\Psi$ of formal groups $G \stackrel{\Psi}{\longrightarrow} F$, defined over a ring $A$, is a series $\psi(u)$ such that $F(\psi(u), \psi(v))=\psi(G(u, v))$. If $\psi(u)=u+O\left(u^{2}\right)$, then $\Psi$ is called a strong isomorphism (invertible change of variables).

The base rings $A$ that had to be considered earlier in fundamental examples were the ring of integers $\mathbf{Z}$, the ring of $p$-adic integers $\mathbf{Z}_{p}$, the residues modulo $p: Z_{p}=\mathbf{Z} / p \mathbf{Z}$, the ring of algebraic integers in some algebraic number-field or its $p$-adic completion. In topology they are rings $\Omega$ of some form of cobordisms, in particular, the ring of unitary cobordisms, which is algebraically isomorphic to the graded ring of polynomials over $\mathbf{Z}$ with polynomial generators of all even degrees.

The reader can find a large collection of examples of formal groups over number rings in Honda's beautiful article [17].

Date: Received by the Editors 3 December 1970.

Translated by C. J. Shaddock. 
Some very simple examples. a) The linear group over $\mathbf{Z}$, where $F_{0}(u, u)=u+v$.

b) The multiplicative group over $\mathbf{Z}$, where $F_{m}(u, v)=u+v \pm u v$; the change of variables $\psi(u)= \pm \ln (1 \pm u)$, which takes the group $F_{m}(u, v)$ into the linear form, lies in the $\operatorname{ring} Q \subset \mathbf{Z}$, so over $\mathbf{Z}$ this group is not isomorphic to the linear group.

c) Lazard's group. Consider the ring $B=\mathbf{Z}\left[x_{1}, \ldots, x_{n}, \ldots\right]$ of integer polynomials in infinitely many variables and the series $g(u)=u+\sum_{n \geq 1} \frac{u^{n+1} x_{n}}{n+1}$. Then there is the group

$$
F(u, v)=g^{-1}(g(u)+g(v))
$$

where $g^{-1}(g(u))=u$. The coefficients $\alpha_{i j}$ of the series $F(u, v)$ lie in the $\operatorname{ring} B \otimes Q$ and generate over $\mathbf{Z}$ a subring $A \subset B \otimes Q$, where $F(u, v)=u+v+\sum_{j \geq 1} \alpha_{i j} u^{i} v^{i}$.

Then the following theorem of Lazard holds.

Theorem 1.1. The ring A of coefficients of the Lazard group is a polynomial ring over $\mathbf{Z}$ with infinitely many generators.

Theorem 1.2. For any commutative one-dimensional formal group over any ring $A^{\prime}$ there exists a unique homomorphism $A \rightarrow A^{\prime}$ under which the Lazard group is mapped into the given group ("universal property of the Lazard group").

Theorem 1.3. For any commutative one-dimensional formal group $F(u, v)$ over any ring $A^{\prime}$ there exists a series $\varphi(u) \in A^{\prime}[[u]] \otimes Q$ such that

$$
\varphi(u)=u+O\left(u^{2}\right) \quad \text { and } \quad F(u, v)=\varphi^{-1}(\varphi(u)+\varphi(v)) \in A^{\prime}[[u, v]] \otimes Q .
$$

Thus, over the rational numbers all groups can be linearized. The series $\varphi(u)$ is called the "logarithm" of the formal group $F(u, v)$. Note that the coefficients of the formal differential $d \varphi(u)=\left(\sum_{n>0} \varphi_{n} u^{n}\right) d u$ lie in $A^{\prime}$, where $\varphi_{0}=1, \varphi(u)=$ $u+\sum_{n \geq 1} \frac{\varphi_{n}}{n+1} u^{n+1}$. The differential $d \varphi$ is called the "invariant differential" of $F(u, v)$ and is calculated thus: $d \varphi=d u /\left(\frac{\partial}{\partial v} F(u, v)\right)_{v=0}$ (see Honda [17]). Over $A^{\prime} \otimes Q$ we also have the equation $\varphi(u)=\left[\frac{1}{k} \varphi^{-1}(k \varphi(u))\right]_{k=0}$.

The proofs of Theorems 1.1-1.3 can be found in [4]; expressions of the form $\frac{1}{k} \varphi^{-1}(k \varphi(u))=\frac{1}{k} F(u, F(u, \ldots) \ldots)=\Psi^{k}(u)$ are connected with the "Adams operators" in topology.

A remarkable fact is that the "formal group of geometric cobordisms", introduced by Mishchenko and S. P. Novikov in [14], which plays an important role and lias a simple geometrical meaning, turns out to coincide with Lazard's universal group. This was first noticed by Quillen in [7] and led to further important applications of this group in topology. The invariant differential in this group has the form $d g(u)=\left(\sum_{n \geq 0}\left[C P^{n}\right] u^{n}\right) d u$, where $\left[C P^{n}\right]$ is the class of unitary cobordisms of complex projective space; the coefficient ring $A$ of the Lazard group coincides with the ring $\Omega$ of unitary cobordisms. Later we shall come across the concept of a "power system", which is weaker than that of formal group.

Definition 1.3. A power system of type $s \geq 1$ over the ring $A$ is a sequence of series $f_{k}(u) \in A[[u]]$ such that $f_{k}(u)=k^{s} u+O\left(u^{2}\right)$ and $f_{k}\left(f_{l}(u)\right)=f_{k l}(u)$, where $k$ and $l$ are any integers (in rings $A$ with torsion it is convenient to require the coefficients of $f_{k}(u)$ to be algebraic with respect to the variable $k$ ).

Then we have a simple fact (Bukhshtaber and Novikov [4]). Over the ring $A \otimes Q$ there exists a series $B(u) \in A[[u]] \otimes Q$ such that $f_{k}(u)=B^{-1}\left(k^{s} B(u)\right)$, where $B^{-1}(B(u))=u$ and $B(u)=u+O\left(u^{2}\right)$. 
Every group generates a power system over the same ring, but the converse is in general not true, since the coefficient ring of a power system is far smaller. The reader can find a number of examples of power systems with their properties and theorems in [4].

Note that in [4], [21] and later sections of this survey we shall meet "two-valued" analogues of formal groups, defined by equations (which do not have solutions over $A[[u, v]])$

$$
Z^{2}-\Theta_{1}(u, v) Z+\Theta_{2}(u, v)=0 ;
$$

here $\Theta_{1}, \Theta_{2}$, can be regarded as the "sum" and "product" of values of the group $F^{ \pm}(u, v)$, not lying in the original ring. The "inverse element" of $u$ is a series $\varphi(u)$ such that $\Theta_{2}(u, \varphi(u))=0$. An important case is that when $\varphi(u)=u($ see $\S 4)$.

\section{$\S 2$. COBORDISM AND BORDISM THEORY}

I. The axiomatics of bordism theory. General properties. Suppose that some class of manifolds, closed and with boundary and possibly with additional structure, is given such that

a) the boundary of a manifold in the class belongs to the class;

b) the direct product of manifolds of the class belongs to the class ( "multiplicative property");

c) a closed domain with smooth boundary in a manifold of the class belongs to the class (and also a closed interval belongs to the class) ("excision axiom" and homotopy invariance).

We say that such a class defines a cobordism (and bordism) theory. We denote the class by $P$.

The cycles (singular bordisms in $P$ ) for any complex $K$ are the pairs $(M, f)$, where $M \in P, f: M \rightarrow K$ is a continuous mapping and $M$ is a closed manifold. The singular "films" are the pairs $(N, g)$, where $N \in P$ has boundary and $g: N \rightarrow K$. In an obvious way, this gives rise to the group of $n$-dimensional cycles factored out by the group of boundaries of films in $P$ for any complex $K$; this group is denoted by $\Omega_{n}^{P}(K)$ and called the "bordism group" of $K$ relative to the class $P$. The group of "relative bordisms" $\Omega_{n}^{P}(K, L)$ is defined similarly and we have the so-called "exact sequence of a pair": $\cdots \rightarrow \Omega_{n}^{P}(L) \rightarrow \Omega_{n}^{P}(K) \rightarrow \Omega_{n}^{P}(K, L) \stackrel{\delta}{\rightarrow} \Omega_{n-1}^{P}(L) \rightarrow \cdots$. A mapping $K_{1} \stackrel{\varphi}{\rightarrow} K_{2}$ induces a homomorphism $\varphi_{*}: \Omega_{n}^{P}\left(K_{1}\right) \rightarrow \Omega_{n}^{P}\left(K_{2}\right)$. The groups $\Omega_{n}^{P}$ and homomorphisms $\varphi_{*}$ are homotopy-invariant (the interval $I^{1} \in P$ ). For Euclidean space $R^{q}$ (or for points), the groups $\Omega_{n}^{P}\left(R^{q}\right)$ are in general non-trivial for $n>0$. The direct sum $\Omega_{*}^{P}=\sum_{n} \Omega_{n}^{P}\left(R^{q}\right)$ forms the "ring of scalars in bordism theory".

For finite complexes $K$ we define the cobordism groups $\Omega_{P}^{n}(K)$, following the Alexander-Pontryagin duality law: if $K \subset S^{N}$, where $S^{n}$ is a sphere and $N$ is large, then by definition we put $\Omega_{P}^{n}(K)=\Omega_{N-n}^{P}\left(S^{N}, S^{N} \backslash K\right)$, and this definition is independent of $N$ and the embedding $K \subset S^{N}$. The groups $\Omega_{P}^{i}$ have the properties of cohomology and the relative groups $\Omega_{P}^{i}(K, L)$ are defined. The sum $\Omega_{P}^{*}=$ $\sum_{n} \Omega_{P}^{n}(K, L)$ forms the "cobordism ring" of the pair: $K \supset L$. For $R^{q}$ (or a point) the ring $\Omega_{P}^{*}=\sum_{n} \Omega_{P}^{n}\left(R^{q}\right)$ is the analogue of the scalars.

For the point $x$ we have $\Omega_{P}^{n}(x)=\Omega_{-n}^{P}(x)$, by definition.

For certain classes of manifolds the "Poincaré-Atiyah duality law" is valid: $D: \Omega_{P}^{i}\left(M^{n}\right) \stackrel{\approx}{\longrightarrow} \Omega_{n-i}^{P}\left(M^{n}\right)$. 
Examples. The most important examples of classes $P$ are connected with the introduction of some kind of structure into the stabilized tangent bundle $\tau_{M}$ of a manifold $M$; for example, orientation in the bundle $\tau_{M} \times R^{k}$ for some $k \geq 0$, complex structure in the bundle $\tau_{M} \times R^{q}$, symplectic structure in $\tau_{M} \times R^{q}$ or trivialization of $\left(-\tau_{M}\right) \times R^{q}$ (framing or the Pontryagin structure), etc. Thus, the classes $P$ of this kind are connected with some class $Q$ of vector bundles over a complex, that is, $P=P(Q)$.

The Thom isomorphism. For classes $P$ connected with classes $Q$ of vector bundles, another property is required in addition to a), b), c) above:

d) the space of a bundle of class $Q$ with fibre a disc and base $M \in P$ is a manifold of class $P$.

If the base space of the bundle $\eta$ is $K$, the bundle space with fibre a disc $D^{n}$ is $E_{\eta}$ and its boundary ${\stackrel{\circ}{E_{\eta}}}_{\eta}$ is a bundle with fibre $S^{n-1}$, then from the definitions and d), the so-called "Thom isomorphism" follows: $\varphi_{P}: \Omega_{i}^{P}(K) \stackrel{\approx}{\longrightarrow} \Omega_{n+i}^{P}\left(E_{\eta}, \stackrel{\circ}{E}_{\eta}\right)$, which is defined by means of the space of the induced fibering $f^{*} \eta$. The Thom isomorphism generates the Poincaré-Atiyah duality for all manifolds of the class $P$ : $D: \Omega_{P}^{i}\left(M^{n}\right) \stackrel{\approx}{\longrightarrow} \Omega_{n-i}^{P}\left(M^{n}\right)$. We can define the fundamental cycle $\left[M^{n}\right] \in \Omega_{n}^{P}\left(M^{n}\right)$, the Cech operation $x \cap y \in \Omega_{n-q}^{P}(K)$ for $x \in \Omega_{P}^{q}, y \in \Omega_{n}^{P}$, and prove that the Poincaré duality is determined by the Čech operation. In addition, for any continuous mapping $f$ we have the identity $f_{*}\left(f^{*} x \cap y\right)=x \cap f_{*} y$, where $x \in \Omega_{P}^{q}$, $y \in \Omega_{n}^{P}$.

II. Unitary cobordisms. The fundamental class $P$ of interest to us is the class of stably quasicomplex manifolds and $Q$ is the class of complex vector bundles. In this case the groups $\Omega_{*}^{P}(K)$ and $\Omega_{P}^{*}(K)$ are denoted, as usual, by $U_{*}(K)$ and $U^{*}(K)$ and are called "unitary bordisms and cobordisms". The $\operatorname{ring} U_{*}($ point $)=\Omega_{*}^{U}$ is the ring of polynomials over $\mathbf{Z}$ in generators of even degree, one in each dimension.

Other bordisms of classes $P$, connected, respectively, with all manifolds, orientable, special unitary, unitnry, stably symplectic or framed manifolds, etc., are denoted, as usual, by $\Omega_{*}^{O}, \Omega_{*}^{S O}, \Omega_{*}^{U}=U_{*}, \Omega_{*}^{S U}, \Omega_{*}^{S p}, \Omega_{*}^{1}=$ (bordisms of framed manifolds). Information on these groups can be found in Novikov's survey [13].

\section{The ring of operations.}

Definition 2.1. A (stable) homology operation is an additive homomorphism $\theta: \Omega_{*}^{P}(K, L) \rightarrow \Omega_{*}^{P}(K, L)$, defined simultaneously for all dimensions and all complexes, that commutes with continuous mappings and also with the boundary homomorphism $\partial: \Omega_{*}^{P}(K, L) \rightarrow \Omega_{*}^{P}(L), K \supset L$.

Such operations form a ring, the "Steenrod ring" $A^{P}$, which is denoted for unitary bordisms $U_{*}$ by $A^{U}$. For cobordisms $U^{*}$, the ring of operations is defined similarly and coincides with the ring of operations $A^{U}$.

If $U_{N}$ is a unitary group, $B U_{N}$ the base of the universal bundle and $\eta_{N}$ the bundle space (with fibre a disc), then $M U$ denotes the spectrum $\left(M U_{N}\right)$ of the Thom spaces $M U_{N}=E_{\eta N} / \stackrel{\circ}{E}_{\eta N}$, where $E_{\eta N}$ is the space of the fibering $\eta_{N}$. The stable homotopy classes of mappings $[K, M U]$ coincide with the ring $U^{*}(K)$. In particular, $A^{U}=$ $[M U, M U]$, and the universal Thom isomorphism $\varphi: U^{*}\left(B U_{N}\right) \stackrel{\approx}{\longrightarrow} U^{*}\left(M U_{N}\right)$ is defined (see [13]). 
Example. Multiplication by a "scalar" $\lambda \in U^{*}$ (point) is evidently a cohomology operation. Note that for $U$-cobordism we have $\Omega_{*}^{U}=\mathbf{Z}\left[x_{1}, \ldots, x_{n}, \ldots\right]$. For cohomology operations in classical homology and cohomology theories the scalars are just ordinary numbers and commute with all the other operations. For cobordisms everything is more complicated.

The ring $A^{U}$ was computed by S.P. Novikov in [14]. It can be described as follows. For any symmetrized decomposition $k=\operatorname{dim} \omega=\sum k_{i}, k_{i} \geq 0$, operators $S_{\omega} \in A^{U}$ are defined such that $S_{(0)}=1$ and any element of $A^{U}$ has the form of a formal series $\sum_{i} \lambda_{i} S_{\omega_{i}}$, where $\operatorname{dim} \omega_{i} \rightarrow \infty$ as $i \rightarrow \infty$ and $\lambda_{i} \in \Omega_{*}^{U}$. Formulae for the composition $S_{\omega_{1}} \circ S_{\omega_{2}}$ were given ${ }^{1}$ in [14] and follow in the end from the Leibniz formula $S_{\omega}(x y)=\sum_{\left(\omega_{1}, \omega_{2}\right)=\omega} S_{\omega_{1}}(x) S_{\omega_{2}}(y)$. A superposition of the form $S_{\omega} \circ \lambda$ is equal to

$$
\lambda \circ S_{\omega}+\sum_{\substack{\left(\omega_{1}, \omega_{2}\right)=\omega \\ \operatorname{dim} \omega_{1}>0}} \sigma_{\omega_{1}}^{*}(\lambda) S_{\omega_{2}}
$$

where the additive homomorphisms $\sigma_{\omega}^{*}(\lambda)$ on $\Omega_{*}^{U}$ are computed by means of the geometry of the manifolds representing $\lambda \in \Omega_{*}^{U}$. For example, $\sigma_{(q)}^{*}\left(\left[C P^{n}\right]\right)=$ $-(n+1)\left[C P^{n-q}\right]$. In particular, the representation ${ }^{*}$ in which $S_{\omega} \stackrel{*}{\rightarrow} \sigma_{\omega}^{*}$ and $\lambda \rightarrow$ (multiplication by $\lambda$ ) of the ring of operations $A^{U}$ on the ring of bordisms of a point $U^{*}$ (point) is faithful.

Geometric bordisms. There are important subsets of "geometric cobordisms" $V(K) \subset U^{2}(K)$ in any complex $K$, or the dual sets $V\left(M^{n}\right) \subset U_{n-2}\left(M^{n}\right)$ for quasicomplex manifolds ("geometric bordisms"), consisting of submanifolds of complex codimension 1. If $u \in V(K)$, then $S_{\omega}(u)=0$ for $\omega \neq(q)$ and $S_{(q)} u=u^{q+1}$. This property completes the axiomatization of the operations $S_{\omega}$ together with the multiplication formula $S_{\omega}(x y)=\sum_{\left(\omega_{1}, \omega_{2}\right)=\omega} S_{\omega_{1}}(x) S_{\omega_{2}}(y)$.

All possible multiplicative operations $\alpha \in A^{U}$, that is, those for which $\alpha(x y)=$ $\alpha(x) \alpha(y)$ for all $x, y \in U^{*}(K)$ and all $K$, are defined by a single series $\alpha(u) \in$ $U^{*}\left(C P^{\infty}\right)$, where $u \in V\left(C P^{\infty}\right)$ and $C P^{\infty}$ is infinite-dimensional complex projective space. It should be said that $U^{*}\left(C P^{\infty}\right)$ is simply the ring of formal serirs $U^{*}\left(C P^{\infty}\right)=\Omega_{U}^{*}[[u]]$, where $\Omega_{U}^{*}=U^{*}$ (point).

Characteristic classes. The formal group. Having the operations $S_{\omega}$ and the Thom isomorphism, we can construct analogues of the "Chern classes" $C_{\omega}(\eta)$ (where $C_{k}=C_{(1, \ldots, 1)}$ by definition) in the usual way ${ }^{2}$ for any $U_{N}$-bundle $\eta$ (see $[8])$. Here $C_{\omega}(\eta) \in U^{*}$ (the base). For $U_{1}$-bundles $\xi$ and $\eta$ the product $\xi \otimes \eta$ is a $U_{1}$-bundle. The class $C_{1}(\xi \otimes \eta)=F\left(C_{1}(\xi), C_{1}(\eta)\right)$ is computed as a formal series with coefficients in $\Omega_{U}^{*}$ (see [14], Appendix 1). This gives rise to the formal group of "geometric cobordisms" $F(u, v)=F\left(C_{1}(\xi), C_{1}(\eta)\right)=C_{1}(\xi \otimes \eta)=u+$ $v-\left[C P^{1}\right] u v+\cdots$. Mishchenko has shown that $F(u, v)=g^{-1}(g(u)+g(v))$, where $g(u)=\sum_{n \geq 0} \frac{C P^{n}}{n+1} u^{n+1}$ and $d g(u)=\left(\sum_{n \geq 0} C P^{n} u^{n}\right) d u=C P(u) d u$.

\footnotetext{
${ }^{1} \mathrm{~A}$ description of the ring $A^{U}$ without a formula for the composition was also obtained by Landweber in [22].

${ }^{2}$ Note that in the theory of cobordism characteristic classes were introduced at the beginning (Conner and Floyd) and on this basis cohomology operations were defined and the computation of the algebra $A^{U}$ given (Novikov).
} 
Formal groups and operations. The analogues of the Adams operations $\Psi^{k} \in$ $A^{U} \otimes \mathbf{Z}\left[\frac{1}{k}\right]$ are defined by the multiplicativity requirement $\Psi^{k}(x y)=\Psi^{k}(x) \Psi^{k}(y)$ and $\Psi^{k}(u)=\frac{1}{k} g^{-1}(k g(u))$ for $u \in V\left(C P^{\infty}\right) \subset U^{2}\left(C P^{\infty}\right)$, that is, are connected with taking $k$-th powers in the formal group $F(u, v)$. They generate a power system. Further, $\Psi^{k} \in A^{U} \otimes Q$ is defined by $\Psi^{0}(u)=g(u)=\left[\frac{1}{k} g^{-1}(k g(u))\right]_{k=0}$ and determines a projection of the cobordism theory $U^{*} \otimes Q$ onto the usual cohomology $H^{*}(; Q)($ see $[14])$.

Generally speaking, there are many multiplicative projectors in the rings $A^{U} \otimes \mathbf{Z}_{p}$ for prime $p$ (see [7], [14]). The canonical projector $\pi_{p}$ was determined by Quillen $([7])$, namely $\pi_{p}^{*}\left[C P^{n}\right]=0$ for $n \neq p^{h}-1$, and $\pi_{p}^{*}\left[C P^{p^{h}-1}\right]=\left[C P^{p^{h}-1}\right]$. Quillen found this projector by starting from the theory of formal groups. The projection operators are important because they single out smaller homology theories that are more convenient, for example, for the computation of homotopy groups by means of the Adams spectral sequence, which was introduced into cobordism theory in [14]. However, we must be able to compute the rings of operations of these smaller theories; here we can use the previously known structure of the ring of operations $A^{U}$ in unitary cobordism theory if the projector is sufficiently simple. Quillen carried out this programme in [7] by finding a successful projector. The role of formal groups in the structure of the theory of operations became obvious; it was also confirmed by results of the authors and Kasparov on fixed points of mappings. Here we should mention especially the results of Mishchenko [11] (see also [4] and $\S 5)$ on manifolds fixed under the action of groups with a non-trivial normal bundle.

Chern characters. We note also that formal groups are closely connected with analogues to the so-called "Chern character". The classical Chern character ch is an additive-multiplicative function of bundles with values in rational cohomology. Novikov showed in [14] that such a function from bundles with values in the cobordism ring is determined by its value on the $U_{1}$-bundle $\eta$, on which it is equal to $\exp (g(u))$, where $u=C_{1}(\eta), g(u)=\sum_{n \geq 0} \frac{C P^{n}}{n+1} u^{n+1}$. Another abstract concept of the Chern character, due to Dold, is not connected with bundles; it is just the isomorphism of theories $c h_{U}: U^{*} \otimes Q \rightarrow H^{*}\left(; \Omega_{U}^{*} \otimes Q\right)$, which is the identity on the homology of a point. Here too the series $g(u)$ appears. As Bukhshtaber [2] has proved, $c h_{U}^{-1}(t)=g(u)$ for a basis element $t \in H^{2}\left(C P^{\infty}\right)$. In [2] he studied the general Chern-Dold character in unitary cobordism and gave several applications, which were developed further in [3], [4] and [21].

III. The Hirzebruch genera. As Novikov has shown in [15], the so-called "multiplicative Hirzebruch genera" $Q(z)$, or homomorphisms $Q: \Omega_{*}^{U} \rightarrow \mathbf{Z}$ such that $Q\left(C P^{n}\right)=\left[Q(z)^{n+1}\right]_{n}=\frac{1}{2 \pi i} \int_{|z|=\varepsilon} \frac{Q^{n+1}}{z^{n+1}} d z$, can be computed in terms of $g^{-1}(z)$; namely $Q(z)=\frac{z}{g_{Q}^{-1}(z)}$, where

$$
g_{Q}(u)=\sum_{n \geq 0} \frac{Q\left(C P^{n}\right)}{n+1} u^{n+1} \quad \text { and } \quad g_{Q}^{-1}\left(g_{Q}(u)\right)=u \text {. }
$$

Thus, all the fundamental concepts and facts of the theory of unitary cohordism, both modern and classical, can he expressed hy means of Lazard's formal group.

Information from $K$-theory. Finally, we stop to consider ordinary complex $K$ theory $K^{*}(X)$, where $K^{i}(X)=K^{i+2}(X)$ for all $i$ (Bott periodicity), $K^{0}(X)$ consists of stable classes of complex bundles over $X$ and $K^{1}(X)$ of homolopy classes of 
mappings $X \rightarrow U_{N}$, where $N>\operatorname{dim} X$. If $\lambda^{i}$ is the exterior power, $\lambda_{i}=\sum_{i \geq 0} \lambda^{i} t^{i}$, then $\lambda_{t}(x+y)=\lambda_{y}(x) \lambda_{t}(y)$ is the exponential operation. For symmetric powers $S_{i}$ we have $S_{t}=\sum_{i \geq 0} S_{i} t^{i}=\frac{1}{\lambda_{t}}$. Further, if $Q_{k}=\sum_{i=1}^{N} t_{i}^{k}(N \rightarrow \infty)$ and $Q_{k}=$ $Q_{k}\left(\sigma_{1}, \ldots, \sigma_{k}\right)$, where $\sigma_{k}\left(t_{1}, \ldots, t_{N}\right)$ are the elementary symmetric functions, then the virtual representation, the so-called Adams operator, is just $Q_{k}\left(\lambda^{1}, \ldots, \lambda^{k}\right)=$ $\Psi^{k}$. It turns out that $\Psi^{k}(x+y)=\Psi^{k}(x)+\Psi^{k}(y), \Psi^{k}(x y)=\Psi^{k}(x) \Psi^{k}(y)$ and $\Psi^{k} \circ \Psi^{l}=\Psi^{k l}$. Further, for the $U_{1}$-bundle $\eta \in K^{0}(X)$ we have $\Psi^{k}(\eta)=\eta^{k}$. The Adams operator $\Psi^{k}$ does not commute with the Bott periodicity operator $\beta: K^{i} \rightarrow K^{i-2}$. We have the formula $\Psi^{k} \cdot \beta=k \beta \cdot \Psi^{k}$. Hence the operators $\Psi^{k}$ are defined in the theory $K^{*} \otimes \mathbf{Z}\left[\frac{1}{k}\right]$. The cohomology of a point in $K$-theory has the form $K^{*}$ (point) $=\mathbf{Z}\left[\beta, \beta^{-1}\right]$ and $\Psi^{k} \beta=k \beta \Psi^{k}$. This completes the description of the operations in $K$-theory. The analogues of "geometric cobordisms" in $K$-theory are the $U_{1}$-bundles; more precisely, they are the elements $u=\beta^{-1}(\xi-1) \in K^{2}(X)$ for the $U_{1}$-bundle $\xi=\beta u+1$. Here $k \Psi^{k}(u)=\beta^{-1}\left((\beta u+1)^{k}-1\right)$, and $F(u, v)=u+$ $v-\beta u v=\beta^{-1}((\beta u+1)(\beta v+1)-1)$ is the multiplicative group. Thus, the well-known Riemann-Roch-Grothendieck homomorphism $r: U^{*}(X) \rightarrow K^{*}(X)$ corresponds to the homomorphism of the universal Lazard group onto the multiplicative group, where $r(\lambda)=T\left(\lambda \cdot \beta^{\frac{\operatorname{dim} \lambda}{2}}\right), T$ is the Todd genus, and $\lambda \in \Omega_{U}^{*}$.

\section{$\S$ 3. The formal group of GeOMETRIC COBORDisms}

The multiplication law in the formal group of geometric cobordisms. Let $\eta \rightarrow C P^{n}, n \leq \infty$, be the canonical complex one-dimensional bundle over the projective space $C P^{n}$. As already noted in $\S 2$, the formal series $c_{1}\left(\eta_{1} \otimes \eta_{2}\right)=F(u, v) \in$ $U^{2}\left(C P^{\infty} \times C P^{\infty}\right)=\Omega_{U}[[u, v]], u=c_{1}\left(\eta_{1}\right), v=c_{1}\left(\eta_{2}\right)$, gives a multiplication law in the one-dimensional formal group of geometric cobordisms over the ring $\Omega_{U}$.

Theorem 3.1. a) The following formula holds:

$$
F(u, v)=\frac{u+v+\sum\left[H_{r, t}\right] u^{r} v^{t}}{C P(u) \cdot C P(v)},
$$

where $H_{r, t}$ the algebraic suhmanifold of complex codimension 1 in $C P^{r} \times C P^{t}$ that consists of the zeros of the sections of the bundle $\eta_{1} \otimes \eta_{2} \rightarrow C P^{r} \times C P^{t}$ and thus realizes the cycle $\left[C P^{r-1} \times C P^{t}+C P^{r} \times C P^{t-1}\right] \in H_{2(r+t-1)}\left(C P^{r} \times C P^{t}\right)$.

b) The logarithm of the group $F(u, v)$ has the form $g(u)=\sum_{n \geq 0} \frac{\left[C P^{n}\right]}{n+1} u^{n+1}$.

Proof. Let $F(u, v)=u+v+\sum e_{i j} u^{i} v^{j}$ and let $\lambda: C P^{r} \times C P^{t} \rightarrow C P^{\infty} \times C P^{\infty}$ be the standard embedding. Then

$$
\epsilon D \lambda^{*} F(u, v)=\left[H_{r, t}\right]=\left[C P^{r-1}\right]\left[C P^{t}\right]+\left[C P^{r}\right]\left[C P^{t-1}\right]+\sum e_{i j}\left[C P^{r-i}\right]\left[C P^{t-j}\right],
$$

where $D$ is the Poincare-Atiyah duality operator, $\epsilon: U^{*}\left(C P^{r} \times C P^{t}\right) \rightarrow \Omega_{U}$ is the augmentation to a point and $\left[C P^{r-i}\right]=\epsilon D U^{i}$ if $u=c_{1}(\eta) \in U^{2}\left(C P^{r}\right)$. Thus, $u+v+\sum\left[H_{r, t}\right] u^{r} v^{t}=F(u, v) C P(u) C P(v)$. This proves a). Now we have the formula

so that

$$
d g(u)=\frac{d u}{\left.\frac{\partial F(u, v)}{\partial v}\right|_{v=0}}
$$

$$
d g(u)=\frac{C P(u) d u}{1+\sum\left(\left[H_{r, 1}\right]-\left[C P^{1}\right]\left[C P^{r-1}\right]\right) u^{r}} .
$$


It is easy to show, for example by comparison of Chern numbers, that $\left[H_{r, 1}\right]=$ $\left[C P^{1}\right]\left[C P^{r-1}\right]$. Hence $d g(u)=C P(u)$. This completes the proof.

The universal property of the formal group of geometric cobordisms. As is shown in [9] and [12], the ring $\Omega_{U}$ is multiplicatively generated by the elements $\left[H_{r, t}\right]$, and according to [16], the ring $\Omega_{U} \otimes Q$ is multiplicatively generated by the elements $\left[C P^{n}\right], n \geq 0$. From Theorem 3.1 we now find that the subring of $\Omega_{U}$ generated by the coefficients of the multiplication law of the formal group of geometric cobordisms coincides with $\Omega_{U}$, and the coefficients of the logarithm of this group are algebraically independent and generate the ring $\Omega_{U} \otimes Q$. We shall now show that from these facts it follows trivially that the group $F(u, v)$ over $\Omega_{U}$ is universal on the category of commutative rings without torsion. Let $G(u, v)$ be any formal group over a ring $R$ without torsion, and let $g_{G}(u)=\sum \frac{a_{n}}{n+1} u^{n+1}, a_{n} \in R$, be its logarithm. We consider the ring homomorphism $r: \Omega_{U} \rightarrow R \otimes Q, r\left[C P^{n}\right]=a_{n}$. Since $G(u, v)=g^{-1}(g(u)+g(v))$, we have $r(F(u, v))=\sum r\left(e_{i j}\right) u^{i} v^{j}=G(u, v)$. Hence $r\left(e_{i j}\right) \in R$, that is, $\operatorname{Im}\left(r: \Omega_{U} \rightarrow R \otimes Q\right) \subset R$. Since the Lazard universal group is defined over a ring without torsion, we have thus proved the theorem.

Theorem 3.2. The formal group of geometric cobordisms coincides with the Lazard universal formal group, that is, the homomorphism of the Lazard ring A into $\Omega_{U}$ corresponding to the group $F(u, v)$ (see $\S 1$ ) is an isomorphism.

The Hirzebruch genera from the point of view of the theory of formal groups. By Theorem 3.2, any integral Hirzebruch genus, or, what is the same thing, a homomorphism $\Omega_{U} \rightarrow \mathbf{Z}$, determines a formal group over $\mathbf{Z}$, and conversely, any formal group over $\mathbf{Z}$ determines a Hirzebruch genus. Here the Hirzebruch genus defining the homomorphism $\Omega_{U} \rightarrow \mathbf{Z}$ may have rational coefficients. Equivalent (strongly isomorphic) formal groups are determined by Hirzebruch series $Q(z)$, $Q^{\prime}(z)$ connected by the formula $\frac{z}{Q(z)}=\varphi^{-1}\left(\frac{z}{Q^{\prime}(z)}\right)$, where $\varphi^{-1}(u)=u+\sum \lambda_{i} u^{i+1}$, $\lambda_{i} \in \mathbf{Z}$. This follows from the fact that the logarithms of the formal groups are equal to $g_{Q}(z)=\left(\frac{z}{Q(z)}\right)^{-1}$, and we have by definition $g_{Q}(z)=g_{Q^{\prime}}(\varphi(z))$. Suppose that we are given an integral Hirzebruch genus, given by a rational series $g_{Q}(u)$. Then the $Q^{\prime}$-genus such that $g_{Q}(u)=g_{Q^{\prime}}(\varphi(u)), \varphi(u)=u+\sum \mu_{i} u^{i+1}, \mu_{i} \in \mathbf{Z}$, also has integer values on $\Omega_{U}$. This is the sense in which Hirzebruch genera are equivalent as formal groups. We shall consider what kinds of formal groups have already been used in topology in connection with the well-known multiplicative genera $c, T, L, A$. Consider the $T_{y}$-genus (see [18]). Since $T_{y}\left(\left[C P^{n}\right]\right)=\sum_{i=0}^{n}(-y)^{i}$, the corresponding formal group over the ring $\mathbf{Z}[[y]]$ has the form $F_{T_{y}}=\frac{u+v+(y-1) u v}{1+u v y}$. For $y=-1,0,1$, we obtain formal groups corresponding to the Euler characteristic $c$, the Todd genus $T$ and the Hirzebruch $L$-genus. For all values of $y$ the uroup $F_{T_{y}}(u, v)$ is equivalent either to a linear group or to a multiplicative group. We now note that the $A$-genus is equivalent as a formal group to the $L$-genus, and we find that up to equivalence all the Hirzebruch genera previously discussed in topology are connected either with linear or with multiplicative groups.

Multiplicative cohomology operations and Hirzebruch genera. Every multiplicative cohomology operation in cobordisms is given uniquely on the one hand by the ring homomorphism $\varphi^{*}: \Omega_{U} \rightarrow \Omega_{U}$ which it induces by the representation in $\S 1$, and on the other hand by its values on the geometric cobordism $u \in U^{2}\left(C P^{\infty}\right)$, that is, by the formal series $\varphi(u)=u+O\left(u^{2}\right) \in U^{2}\left(C P^{\infty}\right)=\Omega_{U}[[u]]$. Note 
that the series $\varphi(u)$ gives a strong isomorphism of the universal group $F(u, v)=$ $u+v+\sum e_{i, j} u^{i} v^{j}$ with the group

$$
\varphi(F(u, v))=u+v+\sum \varphi^{*}\left(e_{i, j}\right) u^{i} v^{j} .
$$

In the theory of characteristic classes ring homomorphisms $\Omega_{U} \rightarrow \Omega_{U}$ are given by "Hirzebruch series" $K(1+u)=Q(u)$,

$$
Q(u)=\frac{u}{a(u)}, \quad a(u)=u+\sum \lambda_{i} u^{i}, \quad \lambda_{i} \in \Omega_{U} \otimes Q .
$$

From the point of view of Hirzebruch series, the action of the series $a(u)$ on the ring $\Omega_{U}$ is given by the formula $a\left(\left[C P^{n}\right]\right)=\left[\left(\frac{u}{a(u)}\right)^{n+1}\right]_{n}$, where $[f(u)]_{n}$ is tne $n$-th coefficient of the series $f(u)$. Thus, every formal series $a(u)=u+O\left(u^{2}\right)$ gives a ring homomorphism $a^{*}: \Omega_{U} \rightarrow \Omega_{U}$ as a multiplicative operation in cobordisms, and also a ring homomorphism $a: \Omega_{U} \rightarrow \Omega_{U}$ defined by the Hirzebruch genus $Q(u)=\frac{u}{a(u)}$. These two actions of the series $a(u)=u+O\left(u^{2}\right)$ on $\Omega_{U}$ do not coincide. For example, for $a(u)=u$ we have $a^{*}\left(\left[C P^{n}\right]\right)=\left[C P^{n}\right], a\left(\left[C P^{n}\right]\right)=0, n>0$. As Bukhshtaber and Novikov have shown in [4], we have ${ }^{1}$

Theorem 3.3. The mapping $g: a(u) \rightarrow a(g(u))$ of the ring of series has the property that $a(u)[x]=a(g(u))^{*}[x]$ for any element $x$, where $g(u)=\sum_{n \geq 0} \frac{\left[C P^{n}\right]}{n+1} u^{n+1}$ is the logarithm of the formal group of geometric cobordisms.

\section{The generalized Todd characteristic class.}

Definition 3.1. The generalized Todd class of a complex bundle $\xi$ over $X$ is the characteristic class $T(\xi) \in H^{*}\left(X, \Omega_{U} \otimes Q\right)$ corresponding to the series $Q(u)=$ $\frac{u}{g^{-1}(u)}, g^{-1}(g(u))=u$.

Consider a continuous mapping $f: M^{2 n} \rightarrow M^{2 m}$ of quasicomplex manifolds and denote by $\tau(f)$ the element $\left(\tau\left(M^{2 n}\right)-f^{*} \tau\left(M^{2 m}\right)\right) \in \tilde{K}\left(M^{2 n}\right)$, where $\tau$ is the tangent bundle.

Theorem 3.4 (see [2]). We have the formula $\operatorname{ch}_{U} D[f]=f ! T(\tau(f))$, where $[f]$ is the bordism class of $f, \mathrm{ch}_{U}$ is the Chern-Dold character (see $\left.\S 2\right)$, and $f_{!}$is the Gysin cohomology homomorphism.

In [2] Bukhshtaber has given various formulae expressing the generalized Todd class $T(\xi)$ in terms of classical characteristic classes of the bundle $\xi$. In a number of cases these formulae make it possible to compute the bordism class of $f$ effectively. We quote the simplest of these formulae.

Theorem 3.5. Let $\eta$ be the one-dimensional bundle over $X$ and $u=c_{1}(\eta) \in$ $H^{2}(X, Z) ;$ then $T(\eta)=\frac{u}{g^{-1}(u)}$ and $g^{-1}(u)=\operatorname{ch}_{U} \sigma_{1}(\eta)=u+\sum\left[M^{2 n}\right] \frac{u^{n+1}}{(n+1) !}$, where $\left[M^{2 n}\right]=\sigma_{1}\left(\xi_{n+1}\right) \in U^{2}\left(S^{2 n+2}\right) \approx \Omega_{U}^{-2 n}$; here $s_{\omega}\left(-\tau\left(M^{2 n}\right)\right)=0, \omega \neq(n)$, $s_{(n)}\left(M^{2 n}\right)=-(n+1) !, \sigma_{1}\left(\xi_{n+1}\right)$ is the first Chern cobordism class of the generator $\xi_{n+1} \in K\left(S^{2 n+2}\right)$ and $s_{\omega}$ is the Chern number corresponding to the decomposition $\omega$.

\footnotetext{
${ }^{1}$ This result in a similar formulation was also obtained by Adams [23].
} 


\section{$\S$ 4. TWO-VALUED FORMAL GROUPS AND POWER SYSTEMS}

The concept of a two-valued formal group. Let $F(u, v)=u+v+\ldots$ be a one-dimensional formal group over a commutative $\operatorname{ring} R$ with unit element 1 , let $\bar{u}=-u+O\left(u^{2}\right) \in R[[u]]$ be the formal series giving the inverse element in the group $F(u, v)$, that is, $F(u, \bar{u})=0$, and let $g_{F}(u)$ be the logarithm of the group $F(u, v)$. In [4] it was shown that the formal series $F(u, v) \cdot F(\bar{u}, \bar{v})+F(u, \bar{v}) \cdot F(\bar{u}, v)=$ $|F(u, v)|^{2}+|F(u, \bar{v})|^{2}$ and $|F(u, v)|^{2} \cdot|F(u, \bar{v})|^{2}$ in the ring $R[[u, v]]$ actually belong to the ring $R[[x, y]] \subset R[[u, v]]$, where $x=u \bar{u}=|u|^{2}, y=|v|^{2}$, that is, have the form $\Theta_{1}(x, y)$ and $\Theta_{2}(x, y)$, respectively. Consider over $R[[x, y]]$ the quadratic equation $\mathcal{Y}[x, y]=Z^{2}-\Theta_{1}(x, y)+\Theta_{2}(x, y)=0$ and let $B(x)=x+O\left(x^{2}\right) \in R[[x]] \otimes Q$ denote the series having the form $g_{F}(u) g_{F}(\bar{u})=-g_{F}^{2}(u)$ in the $R[[u]] \otimes Q \supset R[[x, y]] \otimes Q$. As noted by Novikov in [4], over a ring $R$ without torsion the solutions of the equation $\mathcal{Y}(x, y)=0$ have the form

$$
F^{ \pm}(x, y)=B^{-1}(\sqrt{B(x)} \pm \sqrt{B(y)})^{2}
$$

These solutions are obviously not formal series in $x$ and $y$, but, as (4.1) shows, have a peculiar associativity. Such quadratic equations were called two-valued formal groups in [4].

Two-valued formal groups and symplectic cobordisms. We consider the two-valued formal cobordism group constructed from the formal group of geometric cobordisms. As was shown in [4], the series $B^{-1}(z)$ in (4.1) coincides with the formal series

$$
\operatorname{ch}_{U}(x)=z+\sum_{n=2}^{\infty}\left[N^{4 n-4}\right] \frac{z^{n}}{(2 n !)} \in H^{*}\left(C P^{\infty}, \Omega_{U} \otimes Q\right),
$$

where $z$ is a generator of the group $H^{4}\left(C P^{\infty}, \mathbf{Z}\right)$ and

$$
s_{(2 n-2)}\left[N^{4 n-4}\right]=(-1)^{n} 2 \cdot(2 n) ! \neq 0 .
$$

Theorem 4.1 (see [4]). For any $n \geq 2$, the bordism classes $\left[N^{4 n-4}\right]$ belong to the image of the homomorphism $\Omega_{S p}^{-4 n+4} \rightarrow \Omega_{U}^{-4 n+4}$. For $n \equiv 1 \bmod 2$ the elements $\left[N^{4 n-4}\right] / 2 \in \Omega_{U}$ already belong to the group $\operatorname{Im}\left(\Omega_{S p} \rightarrow \Omega_{U}\right)$.

The canonical mapping of spectra $\omega: M S p \rightarrow M U$ corresponding to the embedding $S p(n) \subset U(2 n)$ defines an epimorphism $A^{U} \rightarrow U^{*}(M S p(n))$ and hence an embedding of the ring $\operatorname{Hom}_{A^{U}}\left(U^{*}(M S p), \Omega_{U}\right)$ in $\Omega_{U}$. We identify $\operatorname{Hom}_{A^{U}}\left(U^{*}(M S p), \Omega_{U}\right)$ with its image in $\Omega_{U}$. There is an embedding $i: \operatorname{Im}\left(\Omega_{S p} \rightarrow \Omega_{U}\right) \subset \operatorname{Hom}_{A^{U}}\left(U^{*}(M S p), \Omega_{U}\right)$, where the homomorpliism $i \otimes Z\left[\frac{1}{2}\right]$ is an isomorphism; this follows easily from [12]. As an addition to Theorem 4.1 we note that the elements $\left[N^{8 n-4}\right] / 2$ belong to the group $\operatorname{Hom}_{A^{U}}\left(U^{*}(M S p), \Omega_{U}\right)$, but do not belong to $\operatorname{Im}\left(\Omega_{S p} \rightarrow \Omega_{U}\right)$ (see [4]). In [4] it was shown that Theorem 4.1 and the properties of the Chern character described in [2] imply the following result:

Theorem 4.2. Let $\Lambda \subset \Omega_{U}$ be the ring generated by the coefficients of the twovalued cobordism formal group:

a) $\Lambda \subset \operatorname{Hom}_{A^{U}}\left(U^{*}(M S p), \Omega_{U}\right)$,

b) $\Lambda\left[\frac{1}{2}\right] \approx \Omega_{S p}^{*}(*) \otimes Z\left[\frac{1}{2}\right]$.

Since the ring $\Lambda$ is essentially smaller than $\Omega_{U}$, there exist many one-dimensional formal groups over $\Omega_{U} \otimes Q$ the squares of whose moduli generate a two-valued formal cobordism group. Bukhshtaber has shown in [21] that the minimal group (in the 
sense of the coefficient ring) among such one-dimensional groups over $\Omega_{U} \otimes Q$ is uniquely determined by the multiplicative projector $\varkappa^{*}: \Omega_{U}\left[\frac{1}{2}\right] \rightarrow \Omega_{U}\left[\frac{1}{2}\right]$, whose value on the geometric cobordism $u \in U^{2}\left(C P^{\infty}\right)$ is equal to $\varkappa(u)=\sqrt{(-u \bar{u})}=$ $u+O\left(u^{2}\right) \in U^{2}\left(C P^{\infty}\right)\left[\frac{1}{2}\right]$. Bukhshtaber has proved [21]:

Theorem 4.3. a) For the element $\sigma \in \Omega_{U}$ to belong to $\operatorname{Hom}_{A^{U}}\left(U^{*}(M S p), \Omega_{U}\right) \subset$ $\Omega_{U}$ it is necessary and sufficient that $\varkappa^{*}(\sigma)=\sigma$.

b) $\operatorname{Hom}_{A^{U}}\left(U^{*}(M S p), \Omega_{U}\right) \cong \operatorname{Im} \varkappa \cap \Omega_{U}$.

Corollary 4.1. The composition of the mappings

$$
S p^{*}(X)\left[\frac{1}{2}\right] \stackrel{\omega}{\longrightarrow} U^{*}(X)\left[\frac{1}{2}\right] \stackrel{\omega}{\rightarrow} \operatorname{Im}\left(\varkappa U^{*}(X)\left[\frac{1}{2}\right]\right)
$$

establishes an isomorphism of the cohomology theory $S p^{*}\left[\frac{1}{2}\right]$ with the theory selected in $U^{*}\left[\frac{1}{2}\right]$ by the projection operator $\varkappa$.

Algebraic properties of two-valued formal groups. Bukhshtaber in [21] has given an axiomatic definition of the two-valued formal group $\mathcal{Y}(x, y)=Z^{2}-$ $\Theta_{1}(x, y) Z+\Theta_{2}(x, y)=0$, including as a special case the quadratic equation determined by the square of the modulus of a one-dimensional formal group. We do not repeat this definition here because it is unwieldy, but only note that in the definition the existence of a formal series $\varphi(x)$ such that $\Theta_{2}(x, \varphi(x))=0$ is required. The series $\varphi(x)$ behaves as an inverse element and plays an important part in the classification of two-valued formal groups. For example, we have

Theorem 4.4. The two-valued formal group of cobordisms over the ring $\Lambda \subset \Omega_{U}$ of coefficients of the series $\Theta_{1}(x, y)$ and $\Theta_{2}(x, y)$ is universal for two-valued groups over rings $R$ without torsion in which $\varphi(x)=x$, that is, $\Theta_{2}(x, x)=0$.

Formal power systems not lying in formal groups. Let $\mathcal{Y}(x, y)=Z^{2}-\Theta_{1}(x, y) Z+$ $\Theta_{2}(x, y)=0$ be the two-valued formal group over the ring $R[[x, y]]$ defined by the square of the modulus of the one-dimensional group $F(u, v) \in R[[u, v]]$. We consider the sequence of formal series $\varphi_{k}(x) \in R[[x]]: \varphi_{0}(x)=0, \varphi_{1}(x)=x$, $\varphi_{2}(x)=\Theta_{1}(x, x), \ldots, \varphi_{n}(x)=\Theta_{1}\left(x, \varphi_{n-1}(x)\right)-\varphi_{n-2}(x), \ldots$ The series $\varphi_{k}(x)=$ $k^{2} x+O\left(x^{2}\right)$, considered in the ring $R[[u]] \supset R[[x]]$, have the form $[u]_{k}[\bar{u}]_{k}$, where $[u]_{k}$ is the $k$-th power of $u$ in the group $F(u, v)$. Hence the sequence of series $\varphi_{k}(x)$ forms a formal power system of type $s=2$. In the case when $R=\Omega_{U}$ and $F(u, v)$ is the formal group of geometric cobordisms, it is easy to show (see [4]) that $\left\{\varphi_{k}(x)\right\}$ is not the system formed by taking powers in any formal group over $\Omega_{U}$. Note that the system $\left\{\varphi_{k}(x)\right\}$ has important topological applications and first appeared implicitly in Novikov's paper [15] on the classification of fixed points under the action of 2-groups of generalized quaternions on quasicomplex manifolds.

In conclusion we show that the construction of the power system $\varphi_{k}(x)$ has a natural generalization. Let $F(u, v)$ be a formal group over a ring $R$ without torsion and let $g_{F}(u)$ be its logarithm. We consider a complete set $\left(\xi_{0}=1, \ldots, \xi_{m-1}\right)$ of $m$-th roots of 1 . Put $B_{m}^{-1}(-y)=\prod_{j=0}^{m-1} g_{F}^{-1}\left(\xi_{j} \sqrt[m]{y}\right), x=\prod_{j=0}^{m-1} g_{F}^{-1}\left(\xi_{j} g_{F}(u)\right)$. Then $-B_{m}(x)=g_{F}(u)^{m}$, and we obtain a formal power system

$$
F_{k}^{(m)}(x)=B_{m}^{-1}\left(k^{m} B_{m}(x)\right)=\prod_{j=0}^{m-1} g_{F}^{-1}\left(k \xi_{j} g_{F}(u)\right)
$$

of type $s=m$. The coefficients of the series $F_{k}^{(m)}(x)$ automatically lie in the ring $R$ for formal groups $F(u, v)$ with complex multiplication by $\xi_{j}$ (raising to the power 
$\left.\xi_{j}\right)$. This construction goes through for the formal group of geometric cohordisms over $\Omega_{U} \otimes \mathbf{Z}_{p}$ and $m=p-1$, where $\mathbf{Z}_{p}$ is the ring of $p$-adic integers. As in the case $m=2$, we can also consider an $m$-valued formal group given by an algebraic equation of degree whose solution is of the form $F(x, y)=B_{m}^{-1}\left(\sqrt[m]{B_{m}(x)}+\sqrt[m]{B_{m}(y)}\right)^{m}$.

\section{$\S 5$. FIXED POINTS OF PERIODIC MAPPINGS IN TERMS OF FORMAL QROUPS}

Conner and Floyd [6] first showed that the language of bordism theory is extremely convenient for the study of fixed points of smooth periodic maps. The use of formal groups enables us to systematize and generalize further the results in this field.

Basic constructions and concepts. Let $M^{n}$ be a smooth quasicomplex closed manifold, $T$ a smooth mapping of $M^{n}$ with $T^{p}=\mathrm{id}, p$ a prime, and suppose that $T$ preserves the quasicomplex structure of $M^{n}$. It is easy to show that the set $X \subset M^{n}$ of fixed points of $T$, that is, points $x \in M^{n}$ such that $T x=x$, forms a disjoint union of finitely many closed submanifolds $N_{i}$ with the natural quasicomplex structure. We can choose tubular neighbourhoods $U_{i}$ of the $N_{i}$ in such a way that the $U_{i}$ are the spaces of the normal bundles of the embeddings of $N_{i}$ in $M^{n}$, and the action of $T$ is linear on $U_{i}$ and free outside the zero sections $N_{i} \subset U_{i}$. Thus, the boundaries of the tubular neighbourhoods $\partial U_{i}$ are quasicomplex manifolds with free action of $Z_{p}$ and so determine a bordism element $\alpha(T)$ of the infinite-dimensional lens space $B Z_{p}, \alpha(T) \in U_{n-1}\left(B Z_{p}\right)$. The element $\alpha(T)$ is determined only by the behaviour of $T$ near the fixed submanifolds $N_{i}$. It is clear that $\alpha(T)=0$ since $\bigcup_{i} \partial U_{i}=\partial\left(M^{n} \backslash \bigcup_{i} U_{i}\right)$ and the action of $T$ on the manifold $M^{n} \backslash \bigcup U_{i}$ is free. Hence the problem of classifying smooth quasicomplex manifolds acted on by $Z_{p}$ in terms of bordisms reduces to the following two questions: a) the description of the action of $Z_{p}$ near the set of fixed points and b) the discovery of sets of fixed submanifolds for which $\alpha(T)=0$.

Statement of the problem. We begin by making precise what we mean by classification of the action of $Z_{p}$ in terms of bordisms. We shall say that a quasicomplex manifold $M^{n}$ acted on by the group $Z_{p}$ is bordant to zero if there exists a quasicomplex manifold with boundary $W$ and a quasicomplex action $T^{\prime}$ on it such that $\left(T^{\prime}\right)^{p}=\mathrm{id}, \partial W=M, T^{\prime} / \partial W=T$. We shall study classes of bordant manifolds in the sense described above. The behaviour of the transformation $T$ near the fixed submanifold is easily described. It is known that if the group $Z_{p}$ acts on a complex bundle $\xi$ leaving the base invariant, then $\xi$ can be represented as a sum $\xi=\bigoplus_{i=1}^{p} \xi_{i}$, and the action of $Z_{p}$ is determined on the bundle $\xi_{i}$ by one of the irreducible unitary representations of $Z_{p}$. Thus, if $T$ is a generator of $Z_{p}, \zeta=\exp \left(\frac{2 \pi i}{p}\right)$, then $T(x)=\zeta^{i} x$ for $x \in \xi_{i}$. In the class of bordant manifolds acted on by $Z_{p}$ the fixed component $N_{i}$ defines a bordism of the sum of $(p-1)$ bundles, that is,

$$
\beta\left(N_{i}\right) \in U_{k_{i}}\left(\prod_{j=1}^{p-1} B U\left(l_{j}^{i}\right)\right) \quad \text { where } \quad \operatorname{dim} N_{i}=k_{i}, \quad \operatorname{dim} \xi_{j}=l_{j}^{i} .
$$

Thus, if $\Omega_{U, p}^{n}$ is the group of $n$-dimensional bordisms acted on by $Z_{p}$, there exists a mapping

$$
\beta: \Omega_{U, p}^{n} \rightarrow \bigoplus_{k+2 \sum l_{i}=n} U_{k}\left(\prod B U\left(l_{i}\right)\right)
$$


associating a manifold acted on by $Z_{p}$ with a set of bordisms defined by the components of the fixed submanifold.

The second problem is whether for any bordism

$$
x \in \bigoplus_{k+2 \sum l_{i}=n} U_{k}\left(\prod B U\left(l_{i}\right)\right)
$$

the element $x$ can be realized as the set of fixed points of some quasicomplex action of $Z_{p}$. As already described, there is a mapping

$$
\alpha: \bigoplus_{k+2 \sum l_{i}=n} U_{k}\left(\prod B U\left(l_{i}\right)\right) \rightarrow U_{n-1}\left(B Z_{p}\right),
$$

where for $\alpha(x)=0$ the element $x$ can be realized as the set of fixed points of an action of $Z_{p}$. In other words, if $A$ is the ring of all bordisms

$$
A=\bigoplus_{\left(k, l_{1}, \ldots, l_{p-1}\right)} U_{k}\left(\prod B U\left(l_{i}\right)\right)
$$

then the sequence $\Omega_{U, p}^{*} \stackrel{\beta}{\rightarrow} A \stackrel{\alpha}{\rightarrow} U_{*}\left(B Z_{p}\right)$ is exact. It is easy to see that $\alpha$ is an epimorphism and $\operatorname{Ker} \beta \approx p \Omega_{U}^{*}$. An interesting case is that of an action of $Z_{p}$, where the fixed submanifold consists only of isolated points or of a submanifold with trivial normal bundle. In the latter case the fixed submanifold is defined by a bordism $x \in \Omega_{U}^{k}$, and a set of weights $x_{1}, \ldots, x_{\frac{1}{2}(n-k)}$ of a representation of $Z_{p}$ in the normal bundle.

Basic formulae. The interesting connections with the formal group in cobordisms are centred around the description of the homomorphism $\alpha$ (for a detailed description see [4]). It is known that the cobordism ring of the space $B Z_{p}$ can be represented in the form

$$
U^{*}\left(B Z_{p}\right)=\Omega_{U}[[u]] / p \Psi^{p}(u)=0 .
$$

For isolated fixed points with a set of weights $\left(x_{1}, \ldots, x_{n}\right)$ we then have the formula obtained by Kasparov [5], Mishchenko [10] and Novikov [15],

$$
\alpha\left(x_{1}, \ldots, x_{n}\right)=\prod_{j=1}^{n} \frac{u}{g^{-1}\left(x_{j} g(u)\right)} \cap \alpha(1, \ldots, 1),
$$

where $g(u)$ is the logarithm of the formal group. It follows from (5.1) that the righthand side of (5.2) makes sense. In the general case the manifolds $C P^{k}$ with the Hopf line-bundle and weight $x$ form a multiplicative basis of $A$ over $\Omega_{U}$. This means that the elements of $A$ are determined by a sequence of numbers $\left(\left(k_{1}, x_{1}\right), \ldots,\left(k_{l}, x_{l}\right)\right)$, $\sum\left(k_{i}+1\right)=n$.

We consider a meromorphic differential $\Omega$ with poles at $z=u$ on the formal group $f(u, v)$, where $\Omega=\Omega(u, z) d z=\frac{d g(z)}{f(u, z)}, \bar{z}=g^{-1}(-g(z))$, invariant with respect to the displacement $u \rightarrow f(u, w), z \rightarrow f(z, w), \Omega \rightarrow \Omega$. This differential is the analogue to $d z /(u-z)$ on the linear group. Let $t=z / u$ and $d t=d z / u$, where $u$ is a parameter. Then $\Omega=\Omega(u, z) d z=G(u, t) d t$, where $G$ has a pole at $t=1$ for all $z$ and $u$. As shown in [11] (see also [4]), we then have the formula

$$
? ? ?=\left[\prod_{q=1}^{l} G\left(g^{-1}\left(x_{q} g(u)\right), t_{q}\right) \frac{u}{g^{-1}\left(x_{q} g(u)\right)}\right]_{k_{1}, \ldots, k_{l}} \cap \alpha_{2 n-1}(1, \ldots, 1),
$$


where []$_{k_{1}, \ldots, k_{l}}$ denotes the coefficient of $t_{1}^{k_{1}} \ldots t_{l}^{k_{l}}$.

The connection with the Atiyah-Bott formula. Apart from the description of admissible fixed-point sets it is interesting to ask in what manifolds these admissible fixed-point sets can be realized, that is, to describe the mapping $\operatorname{Ker} \alpha \rightarrow \Omega_{U} \otimes$ $Z / p Z$. It turns out that the permissible set

$$
\left(x_{1}, \ldots, x_{n}\right)-\prod_{j=1}^{n} \frac{u}{x_{j} \Psi^{x_{j}}(u)} \cap\left(1_{1}, \ldots, 1_{n}\right),
$$

where

$$
u^{k} \cap\left(1_{1}, \ldots, 1_{n}\right)=\left(1_{1}, \ldots 1_{n-k}\right),
$$

can be realized on a manifold of class

$$
\left[\prod_{j=1}^{n} \frac{u}{x_{j} \Psi^{x_{j}}(u)}\right]_{n} \in \Omega_{U}^{2 n} \otimes Z / p Z
$$

From Atiyah and Bott's paper [1] we can extract the following formula for the Todd genus of $M^{n}$ in terms of the weiglits of the mapping at the fixed points

$$
-T\left(M^{n}\right) \equiv \sum_{j} \operatorname{Tr}\left(\prod_{k=1}^{n}\left(1-\exp \left(-\frac{2 \pi i x_{k}^{j}}{p}\right)\right)^{-1}\right) \bmod p
$$

where $\operatorname{Tr}: Q(\sqrt[p]{1}) \rightarrow Q$ is the number-theoretic trace and the summation in (5.3) is taken over all fixed points. It was of interest to obtain a result for cobordisms similar to that of Atiyah and Bott. This problem is connected with the construction of a homomorphism $\gamma: A \rightarrow \Omega_{U} \otimes Q_{p}$ that coincides with $\prod_{j} \frac{u}{x_{j} \Psi^{x_{j}}(u)}$ on $\operatorname{Ker} \alpha$. Here we understand by the ring $A$ only the fixed submanifold with trivial bundle. As shown in [4], the formula for the homomorphism $\gamma$ has the form

$$
\gamma\left(x_{1}, \ldots, x_{n}\right)=\left[\frac{1}{x_{1} \cdots x_{n}}\left(\prod_{j=1}^{n} \frac{u}{\Psi^{x_{j}}(u)}\right) \frac{u}{\Psi^{p}(u)}\right]_{n} .
$$

Applying the Todd genus $F: \Omega_{U} \rightarrow Z$ to (5.4), we obtain a numerical function

$$
\gamma\left(x_{1}, \ldots, x_{n}\right)=\left[\frac{p u}{1-(1-u)^{p}} \prod_{k=1}^{n} \frac{u}{1-(1-u)^{x_{k}}}\right]_{n} .
$$

However, it does not coincide with the Atiyah and Bott function

$$
A B\left(x_{1}, \ldots, x_{n}\right)=\operatorname{Tr}\left(\prod_{k=1}^{n}\left(1-\exp \left(-\frac{2 \pi i x_{k}}{p}\right)\right)^{-1}\right) .
$$

The functions (5.5) and (5.6) coincide only on Ker $\alpha$. More precisely, let $K \Phi\left(x_{1}, \ldots, x_{n}\right)_{m}$, $0 \leq m \leq n-1$, be the composition of the function

$$
\left[\frac{u}{\Psi^{p}(u)} \prod_{k=1}^{n} \frac{u}{x_{k} \Psi^{x_{k}}(u)}\right]_{m}
$$

with the Todd genus. Note that for an admissible set of fixed points (5.7) vanishes.

Theorem 5.1 (see [4]).

$$
A B\left(x_{1}, \ldots, x_{n}\right)=\gamma\left(x_{1}, \ldots, x_{n}\right)+\sum_{m=0}^{n-1} K \Phi\left(x_{1}, \ldots, x_{n}\right)_{m} \bmod p \mathbf{Z} p
$$


It follows from Theorem 5.1 that Atiyah and Bott's results on the calculation of the Todd genus as an invariant of the fixed points is a reduction of the analogous problem in cobordisms by means of the Todd genus. It is of interest to note, as Faddeev has pointed out, that the Atiyah-Bott formula can be expressed in terms of the formal group corresponding to the multiplicative homomorphism $T: \Omega_{U} \rightarrow Z$, the so-called multiplicative formal group. Namely (see [4])

$$
A B\left(x_{1}, \ldots, x_{n}\right)=\sum_{m=0}^{n}\left[\frac{p u}{\langle u\rangle_{p}} \prod_{k=1}^{n} \frac{u}{\langle u\rangle_{x_{k}}}\right]_{m}=-\left[\frac{p\langle u\rangle_{p-1}}{\langle u\rangle_{p}} \prod_{k=1}^{n} \frac{u}{\langle u\rangle_{x_{k}}}\right]_{m} \bmod p \mathbf{Z} p,
$$

where $\langle u\rangle_{q}$ is the $q$-th power of $u$ in the formal group $f(u, v)=u+u-u v$.

The action of the circle on quasicomplex manifolds. Gusein-Zade has recently studied the fixed points under the action of the circle $S^{1}$ on quasicomplex manifolds. As in the case of $Z_{p}$, we can construct the Conner-Floyd exact sequence $0 \rightarrow U_{*}\left(S^{1}\right) \stackrel{\gamma}{\rightarrow} \bigoplus U_{*}\left(\prod B U\left(n_{i}\right)\right) \stackrel{\alpha}{\rightarrow} U_{*}\left(S^{1},\left\{Z_{s}\right\}_{s}\right) \rightarrow 0$, where the middle term describes the structure of the action of $S^{1}$ near the fixed points and the last term denotes the bordism group of the action of $S^{1}$ without fixed points (stationary points are allowed). Gusein-Zade's remarkable result consists of the description of the last term of this sequence. Namely

$$
U_{*}\left(S^{1},\left\{Z_{s}\right\}_{s}\right) \approx \bigoplus U_{*}\left(\prod_{i} B U\left(n_{i}\right) \times B U(1)\right) .
$$

After (5.8) is established, the description of the homomorphism $\alpha$ is easily reduced to an algebraic problem using the language of formal groups. Because of their complexity we do not give the formulae here. (See the account of Gusein-Zade's results in [21].)

\section{Appendix I ${ }^{1}$. Steenrod POWERs in COBORdisms AND A NEW Method OF COMPUTING THE BORDISM RING OF QUASICOMPLEX MANIFOLDS ${ }^{2}$}

The Thom isomorphisms in cobordisms. For any complex bundle $\xi$ over $X$, $\operatorname{dim} \xi=n$, the universal Thom class $u(\xi) \in U^{2 n}(M(\xi))$ is defined, corresponding to the classifying map $M(\xi) \rightarrow M U(n)$, where $M(\xi)$ is the Thom complex of $\xi$. Multiplication by the element $u(\xi)$ determines the functorial Thom isomorphism $\varphi(\xi): U^{q}(X) \rightarrow \tilde{U}^{q+2 n}(M(\xi)), \varphi(\xi)(\alpha)=u(\xi) \alpha$. Consider a pair of complexes $i: Y \subset X$, and let $\xi^{\prime}$ denote the restriction of $\xi$ to $Y$. The following homomorphism is defined:

$$
\varphi\left(\xi, \xi^{\prime}\right): \tilde{U}^{q}(X / Y) \rightarrow \tilde{U}^{q+2 n}\left(M(\xi) / M\left(\xi^{\prime}\right)\right), \quad \varphi\left(\xi, \xi^{\prime}\right)(\alpha)=u(\xi) \alpha .
$$

Since $i^{*} u(\xi)=u\left(\xi^{\prime}\right)$ and $\varphi(\xi), \varphi\left(\xi^{\prime}\right)$ are isomorphisms, $\varphi(\xi, \xi)$ is an isomorphism. Let $\xi$ and $\eta$ be bundles over $X$. Consider the composition of mappings

$$
\begin{aligned}
\Delta: M(\xi+\eta) / M\left(\xi^{\prime}+\eta^{\prime}\right) & \stackrel{j}{\rightarrow} M(\xi \times \eta) / M\left(\xi^{\prime} \times \eta^{\prime}\right) \stackrel{\approx}{\rightarrow} \\
& \stackrel{\approx}{\longrightarrow}(M(\xi) \wedge M(\eta)) /\left(M\left(\xi^{\prime}\right) \wedge M\left(\eta^{\prime}\right)\right) \rightarrow M(\xi) \wedge\left(M(\eta) / M\left(\eta^{\prime}\right)\right),
\end{aligned}
$$

\footnotetext{
${ }^{1}$ The appendix was written by Bukhshtaber on work of Dieck [19] and Quillen [20].

${ }^{2}$ The bordism ring of quasicomplex manifolds has already been computed (Milnor, Novikov) by using the Adams spectral sequence. The aim of the new method proposed by Quillen is to avoid using this sequence.
} 
where $M(\xi \times \eta)$ is the Thom complex of the bundle $\xi \times \eta$ over $X \times X$, the mapping $j$ is defined by the diagonal $X \rightarrow X \times X$ and $(X \times Y) / X \times * \cup * \times Y=X \wedge Y$, where $*$ is a distinguished point. The following homomorphism is defined:

$$
\begin{aligned}
\Phi(\xi): \tilde{U}^{q}\left(M(\eta) / M\left(\eta^{\prime}\right)\right) & \rightarrow \tilde{U}^{q+2 n}\left(M(\xi+\eta) / M\left(\xi^{\prime}+\eta^{\prime}\right)\right) \\
\Phi(\xi) \alpha & =\Delta^{*}(u(\xi) \cdot \alpha) .
\end{aligned}
$$

Since $u(\xi \times \eta)=u(\xi) \cdot u(\eta) \in U^{*}(M(\xi \times \eta))$, we have $\Phi(\xi) \varphi(\eta)=\varphi\left(\xi+\eta, \xi^{\prime}+\eta^{\prime}\right)$, so that $\Phi(\xi)$ is an isomorphism.

Steenrod exterior powers. Let $S^{\infty}=\lim S^{2 n+1}$ be the infinite-dimensional sphere and $S^{\infty} \rightarrow B Z_{p}=L_{p}^{\infty}$ be the universal $Z_{p}$-bundle. For any $X$ with distinguished point $*$, let $E(X)$ denote the space $\left(S^{\infty} \cup *\right) \wedge \underbrace{X \wedge \cdots \wedge X}_{p \text { times }}$. There is a canonical action of the group $Z_{p}$ defined on $E(x)$ whose restriction to $X \wedge \cdots \wedge X$ is represented by a permutation of the factors. Put $E_{p}(X)=E(X) / Z_{p}$. The correspondence $X \mapsto E_{p}(X)$ is obviously functorial with respect to mappings $X \rightarrow Y$. Consider the bundle $\xi \times \cdots \times \xi$ over the complex $V=S^{\infty} \times X \times \cdots \times X$ lifted from $X \times \cdots \times X$. Since $Z_{p}$ acts freely on $V$, the bundle $\xi_{(p)}=(\xi \times \cdots \times \xi) / Z_{p} \rightarrow V / Z_{p}$ is defined. We have the equation $E_{p}(M(\xi))=M\left(\xi_{(p)}\right)$.

Definition I.1. By an exterior Steenrod power in $U$-cobordism we mean a collection $P_{e}=\left\{P_{e}^{2 n}, n \in \mathbf{Z}\right\}$ of natural mappings $P_{e}^{2 n}: \tilde{U}^{2 n}(X) \rightarrow \tilde{U}^{2 n p}\left(E_{p}(X)\right)$, such that:

1) $i^{*} P_{e}^{2 n}(a)=a^{p} \in \tilde{U}^{2 n p}(X \wedge \cdots \wedge X)$, where $i: X \wedge \cdots \wedge X \rightarrow E_{p}(X)$, $i\left(x_{1}, \ldots, x_{p}\right)=\left(e, x_{1}, \ldots, x_{p}\right), e \in S^{\infty}$ is the embedding;

2) $P_{e}^{2(n+m)}(a b)=T^{*}\left(P_{e}^{2 n}(a) P_{e}^{2 m}(b)\right) \in \tilde{U}^{2(n+m) p}\left(E_{p}(X \wedge Y)\right)$, where $a \in \tilde{U}^{2 n}(X)$, $b \in \tilde{U}^{2 m}(Y), a b \in \tilde{U}^{2(n+m)}(X \wedge Y)$ and $T: E_{p}(X \wedge Y) \rightarrow E_{p}(X) \wedge E_{p}(Y)$, $T\left(e, x_{1}, y_{1}, \ldots, x_{p}, y_{p}\right)=\left(e, x_{1}, \ldots, x_{p}, e, y_{1}, \ldots, y_{n}\right)$;

3) $P_{e}^{2 n}(u(\xi))=u\left(\xi_{(p)}\right) \in \tilde{U}^{2 n p}\left(M\left(\xi_{(p)}\right)\right)$, where $\xi$ is a bundle over $X, \operatorname{dim} X=n$.

It follows from the axioms that for the canonical element $u_{n} \in U^{2 n}(M U(n))$ we have the formula $P_{e}^{2 n} u_{n}=u\left(\eta_{n,(p)}\right)$, where $\eta_{n}$ is the universal $U(n)$-bundle over $B U(n)$. Now let the element $e \in \tilde{U}^{2 n}(X)$ be represented by the mapping $f: S^{2 k} X \rightarrow M U(k+n)$. Since $S^{2 k} X=M(k) / M\left(k^{\prime}\right)$, where $k$ is the trivial $k$-dimensional bundle over $X$ and $k^{\prime}$ is its restriction to $* \in X$, we see that $E_{p}\left(S^{2 k} X\right)=M\left(k_{(p)}\right) / M\left(k_{(p)}^{\prime}\right)$, where $k_{(p)}^{\prime}$ is the restriction of the bundle $k_{(p)}$ to the subcomplex $Y \subset\left(S^{\infty} \times X \times \cdots \times X\right) / Z_{p}$ formed by points $\left(e, x_{1}, \ldots, x_{p}\right)$ in which at least one coordinate $x_{i}=* \in X$. Since $E_{p}(X)=M(0) / M\left(0^{\prime}\right)$ the mapping $\Delta: E_{p}\left(S^{2 k} X\right)=M\left(k_{(p)}\right) / M\left(k_{(p)}^{\prime}\right) \rightarrow E_{p}(X) \wedge E_{p}\left(S^{2 k} X\right)$ is defined and induces an isomorphism $\Phi\left(k_{(p)}\right): U^{*}\left(E_{p}(X)\right) \rightarrow U^{*}\left(E_{p}\left(S^{2 k} X\right)\right), \Phi\left(k_{(p)}\right)(a)=\Delta^{*}\left(u\left(k_{(p)}\right) \cdot a\right)$.

Since $f^{*} u_{k+n}=u(k) \cdot a$, we get $E_{p}(f)^{*}\left(u\left(\eta_{k+n,(p)}\right)\right)=\Phi\left(k_{(p)}\right)\left(P_{e}^{2 n} a\right)$. From the properties of the isomorphism $\Phi\left(k_{(p)}\right)$ it easily follows that this formula uniquely determines an element $P_{e}^{2 n}(a) \in \tilde{U}^{2 n p}\left(E_{p}(X)\right)$. Thus, the exterior Steenrod powers in cobordism exist and are unique.

Steenrod powers in cobordism. The diagonal map $X \rightarrow X \wedge \cdots \wedge X$ defines an embedding $i:\left(L_{p}^{\infty} \cup *\right) \wedge X=\left(S^{\infty} \cup *\right) \wedge Z / Z_{p} \rightarrow E_{p}(X)$.

Definition I.2. By a Steenrod power we mean a collection of natural mappings $P=\left\{P^{2 n}: \tilde{U}^{2 n}(X) \rightarrow \tilde{U}^{2 n p}\left(\left(L_{p}^{\infty} \cup *\right) \wedge X\right), n \in Z\right\}$ such that $P^{2 n}(a)=i^{*} P_{e}^{2 n} a$. 
Let $j: B U(n) \rightarrow B U(n) \times \cdots \times B U(n)$ be the diagonal map. The embedding $i:\left(L_{p}^{\infty} \cup *\right) \wedge M U(n) \rightarrow E_{p}(M U(n))$ decomposes into the composition $\left(L_{p}^{\infty} \cup *\right) \wedge$ $M U(n) \stackrel{\lambda}{\rightarrow} M\left(\left(j^{*} \eta_{n}\right)_{(p)}\right) \stackrel{\vec{j}}{\rightarrow} M\left(\eta_{n,(p)}\right)=E_{p}(M U(n))$. Let $C^{p}$ be $p$-dimensional complex linear space on which $Z_{p}$ acts by permuting coordinates. Consider the complex bundle $\tilde{v}=S^{\infty} \times_{Z_{p}} C^{p} \rightarrow L_{p}^{\infty}$. It follows immediately that $M\left(\left(j^{*} \eta_{n}\right)_{(p)}\right)$ is the Thom space of the bundle $\tilde{v} \otimes \eta_{n} \rightarrow L_{p}^{\infty} \times B U(n)$. We shall compute the Chern class $\sigma_{n p}\left(\tilde{v} \otimes \eta_{n}\right) \in U^{2 n p}\left(L_{p}^{\infty} \times B U(n)\right)$. Splitting the representation of $Z_{p}$ in $C^{p}$ into a sum of one-dimensional representations, we find that $\tilde{v}$ is isomorphic to the sum of bundles $1+\sum_{q=1}^{p-1} \eta^{q}$, where $\eta$ is the canonical bundle over $L_{p}^{\infty}$. Next we represent $\eta_{n}$ in the form of a sum of formal one-dimensional bundles $\sum_{l=1}^{n} \mu_{l}$ :

$$
\begin{aligned}
& \sigma_{n p}\left(\tilde{v} \otimes \eta_{n}\right)= \\
& \prod_{l=1}^{n} \prod_{q=0}^{p-1} \sigma_{1}\left(\eta^{q} \otimes \mu_{l}\right)=\prod_{l=1}^{n} \prod_{q=0}^{p-1}\left(\sigma_{1}\left(\eta^{q}\right)+\sigma_{1}\left(\mu_{l}\right)+\sum e_{i, j} \sigma_{1}\left(\eta^{q}\right)^{i} \sigma_{1}\left(\mu_{l}\right)^{j}\right),
\end{aligned}
$$

where $e_{i, j} \in \Omega_{U}^{-2(i+j-1)}$ are the coefficients of the formal group of geometric cobordisms. We denote the ring generated by the elements $e_{i, j}$ by $A \subset \Omega_{U}$. Since the elements $\sigma_{1}\left(\eta^{q}\right) \in U^{2}\left(L_{p}^{\infty}\right)$ are represented by formal series in $u=\sigma_{1}(\eta)$ with coefficients from the subring $A \subset \Omega_{U}$, we have

$$
\sigma_{n p}\left(\tilde{v} \otimes \eta_{n}\right)=\sigma_{n}\left(\eta_{n}\right)\left(w^{n}+\sigma_{n}\left(\eta_{n}\right)^{p-1}+\sum w^{n-|\omega|} \alpha_{\omega}(u) \sigma_{\omega}\left(\eta_{n}\right)\right)
$$

where $w=\sigma_{p-1}\left(\sum_{q=1}^{p-1} \eta^{q}\right), \sigma_{\omega}\left(\eta_{n}\right)$ is the characteristic class corresponding to the partition $\omega=\left(i_{1}, \ldots, i_{n}\right),|\omega|=\sum i_{k}$ and $\alpha_{\omega}(u) \in U^{*}\left(L_{p}^{\infty}\right)$ is a polynomial in $u=\sigma_{i}(\eta)$ with coefficients in $A$. We now note that the space $\left(L_{p}^{\infty} \cup *\right) \wedge M U(n)$ is the Thom complex of the bundle $\eta_{n} \rightarrow L_{p}^{\infty} B U(n)$ in which the mapping of Thom complexes $\lambda:\left(L_{p}^{\infty} \cup *\right) \wedge M U(n) \rightarrow M L\left(\left(j^{*} \eta_{n}\right)_{(p)}\right)$ is the identity on the base. Recall that the cohomology operations $S_{\omega}\left(u_{n}\right)$ can be defined by the formula $S_{\omega}\left(u_{n}\right)=$ $u_{n} \cdot \sigma_{\omega}\left(\eta_{n}\right)$. We have $P^{2 n} u_{n}=i^{*} P_{e}^{2 n} u_{n}=\lambda^{*} j^{*} u\left(\eta_{n,(p)}\right)=\lambda^{*} u\left(\left(j^{*} \eta_{n}\right)_{(p)}\right)=w^{n} u_{n}+$ $u_{n}^{p}+\sum w^{n-|\omega|} \alpha_{\omega}(u) S_{\omega}\left(u_{n}\right)$. Here we use the fact that if we restrict the Thom class $u(\xi)$ to the zero section of the bundle $\xi$, it becomes by definition the characteristic class $\sigma_{n}(\xi)$, where $n=\operatorname{dim} \xi$.

Theorem I.1. Suppose that the element $a \in U^{2 n}(X)$ is represented by the mapping $f: S^{2 k} X \rightarrow M U(k+n)$; then in the ring $U^{*}\left(L_{p}^{\infty} \times X\right)$ we have the formula

$$
w^{k} P^{2 n} a=w^{n+k} a+\sum w^{n-|\omega|} \alpha_{\omega}(u) S_{\omega}(a) .
$$

where $w=\sigma_{p-1}\left(\sum_{q=1}^{p-1} \eta^{q}\right) \in U^{*}\left(L_{p}^{\infty}\right)$ and $\alpha_{\omega}(u) \in U^{*}\left(L_{p}^{\infty}\right)$ are polynomials in $u$ with coefficients in $A$, the ring generated by the coefficients of the multiplication law of the formal group of geometric cobordisms.

Proof. Let $u(k) \in \tilde{U}^{2 k}\left(S^{2 k}\right)=Z$ be a generator. Then $f^{*} u_{k+n}=u(k) \cdot a$. Hence $f^{*} P^{2(k+n)} u_{k+n}=T^{*}\left(P^{2 k} u(k) \cdot P^{2 n}(a)\right)$, where $T:\left(L_{p}^{\infty} \cup *\right) \wedge S^{2 k} X \rightarrow\left(L^{\infty} \cup *\right) \wedge$ $S^{2 k} \wedge\left(L_{p}^{\infty} \cup *\right) \wedge X$. The element $u(k)$ is represented by the inclusion $S^{2 k} \subset M U(k)$, so that $P^{2 k} u(k)=w^{k} u(k)$. If we now use the formula for the element $P^{2(k+n)} u_{k+n}$, we obtain the proof of the theorem. 
Computation of the bordism ring of quasicomplex manifolds. Standard arguments of homotopy theory not using information about the ring $\Omega_{U}$ show that if the canonical mapping $\mu: U^{*}(X) \rightarrow H^{*}(X, Z)$ is an epimorphism and the group $H^{*}(X, Z)$ has no torsion, then the group $U^{*}(X)$ is a free $\Omega_{U}$-module (see [8], Appendix). Since the construction of characteristic classes $\sigma_{\omega}(\xi)$ in cobordism can also be carried out independently of results on the ring $\Omega_{U}$, and in addition, $\mu \sigma_{\omega}(\xi)=c_{\omega}(\xi) \in H^{2|\omega|}(X, Z)$, where $c_{\omega}$ are the classical Chern classes (see [6], Appendix), we find that the groups $U^{*}(B U(n) \times B U(k))$ and $U^{*}(M U(n)), n \geq 1$, $k \geq 1$, are free $\Omega_{U}$-modules. In particular, $U^{*}\left(C P^{\infty} \times C P^{\infty}\right)=\Omega_{U}[[u, v]]$, where $u, v$ are the first Chern cobordism classes of the canonical one-dimensional bundles $\eta_{1}$ and $\eta_{2}$. Let $A=\mathbf{Z}\left[y_{1}, \ldots, y_{2}\right]$ be the universal Lazard group and $\varphi: A \rightarrow \Omega_{U}$ the ring homomorphism corresponding to the formal group of geometric cobordisms

$$
F(u, v)=\sigma_{1}\left(\eta_{1} \otimes \eta_{2}\right) \in U^{2}\left(C P^{\infty} \times C P^{\infty}\right) .
$$

In $\S 3$ we showed by direct computation that the coefficients of the logarithm $g(u)$ of the group $F(u, v)$ are algebraically independent. But since the coefficients of the logarithm of the Lazard group generate the $\operatorname{ring} A \otimes Q$, we see that $\varphi$ is a monomorphism.

Consider the formal series $\Theta_{p}(u)=\frac{[u]_{p}}{u}=p+\alpha_{1} u+\ldots$ over $\Omega_{U}[[u]]$, where $[u]_{p}$ is the $p$-th power of $u$ in the formal group.

Lemma I.1. There is an exact sequence

$$
\Omega_{U} \stackrel{\Theta_{p}(u)}{\longrightarrow} U^{q}\left(L_{p}^{\infty}\right) \stackrel{u}{\rightarrow} U^{q+2}\left(L_{p}^{\infty}\right),
$$

where $u=\sigma_{1}(\eta), \eta$ is the canonical bundle over $L_{p}^{\infty}$, and the homomorphisms in the sequence are multiplications by $\Theta_{p}(u)$ and $u$.

Proof. The symbol $\eta$ always denotes the canonical bundle over $C P^{\infty}$. Consider the bundle $\eta^{p} \rightarrow C P^{\infty}$, and denote by $E \rightarrow C P^{\infty}$ the bundle with fibre a disc $D^{2}$ associated with $\eta^{p}$. Then $\partial E=L_{p}^{\infty}, E / \partial E=M\left(\eta^{p}\right)$. By considering the homomorphism $\mu: U^{*}\left(L_{p}^{\infty}\right) \rightarrow H^{*}\left(L_{p}^{\infty}, Z\right)$, it follows that the homomorphism $U^{*}(E)=U^{*}\left(C P^{\infty}\right) \rightarrow U^{*}\left(L_{p}^{\infty}\right)$ is an epimorphism, and since $\sigma_{1}\left(\eta^{p}\right)=[u]_{p}$, we find that there is an exact sequence $0 \leftarrow U^{*}\left(L_{p}^{\infty}\right) \leftarrow U^{*}\left(C P^{\infty}\right) \stackrel{[u]_{p}}{\longleftarrow} \tilde{U}^{*}\left(M\left(\eta^{p}\right)\right) \leftarrow 0$. The lemma now follows because the homomorphism of multiplication by $u$ in the ring $U^{*}\left(C P^{\infty}\right)=\Omega_{U}[[u]]$ is a monomorphism.

Theorem I.2. The homomorphism $\varphi: \mathbf{Z}\left[y_{1}, \ldots, y_{n}, \ldots\right] \rightarrow \Omega_{U}$ of the Lazard group into $\Omega_{U}$ corresponding to the formal group of geometric cobordisms is an isomorphism.

Proof. We only have to prove that $\varphi$ is an epimorphism. We put $C=\operatorname{Im} \varphi \subset \Omega_{U}$ and prove that for any $n \geq 0$ there is an isomorphism $U^{*}\left(S^{n}\right)=C \sum_{q \geq 0} U^{q}\left(S^{n}\right)$. We note first that because of the isomorphism $U^{q}\left(S^{n}\right) \simeq U^{q+1}\left(S^{n+1}\right)$ and because $U^{q}\left(S^{n}\right)$ is finitely generated for each $q$, it is sufficient to prove that for any prime $p$ there is an isomorphism $\tilde{U}^{e v}\left(S^{n}\right) \otimes Z_{p}=C \cdot \sum_{q>0} U^{2 q}\left(S^{n}\right) \otimes Z_{p}$. Put $R_{p}=$ $C \cdot \sum_{q>0} U^{2 q}\left(S^{n}\right) \otimes Z_{p}$. Assume that for all $j<q$ we have already proved the isomorphism $R_{p}^{-2 j}=\tilde{U}^{-2 j}\left(S^{n}\right) \otimes Z_{p}$. For $q=0$ this isomorphism is obvious. Let the element $a \in \tilde{U}^{-2 q}\left(S^{n}\right)$ be represented by the mapping $f: S^{2 k} S^{n} \rightarrow M U(k-q)$; 
then by Theorem I.1 we have the formula

$$
w^{k} P^{-2 q} a=w^{k-q} a+\sum w^{n-|\omega|} \alpha_{\omega}(u) \cdot S_{\omega}(a) .
$$

The element $w \in U^{2 n p}\left(L_{p}^{\infty}\right)$ is represented by a formal scries of the form $(p-1) ! u^{p-1}+O\left(u^{p}\right)$ with coefficients in the ring $C$. By the inductive hypothesis $S_{\omega}(a) \in R_{p},|\omega|>0$, and from (I.2) we have for any $m$ the formula

$$
u^{m}\left(w^{q} P^{-2 q} a-a\right)=\psi(u) \in U^{*}\left(L_{p}^{\infty} \times S^{\infty}\right) \approx U^{*}\left(L_{p}^{\infty}\right) \otimes_{\Omega_{U}} U^{*}\left(S^{n}\right),
$$

where $\psi(u) \in R_{p}[[u]]$. We assume that $m \geq 1$ is the smallest of the numbers for which (I.3) holds. Since $\psi(0)=0$, we have $\psi(u)=u \psi_{1}(u), \psi_{1}(u) \in R_{p}[[u]]$, and we obtain the formula $u\left(u^{m-1}\left(w^{q} P^{-2 q} a-a\right)-\psi_{1}(u)\right)=0$. Then by Lemma I.1 there exists an element $y \in U^{*}\left(S^{q}\right)$ such that

$$
u^{m-1}\left(w^{q} P^{-2 a} a-a\right)=\psi_{1}(u)+y \Theta_{p}(u) \in U^{*}\left(L_{p}^{\infty} \times S^{n}\right) .
$$

Considering the restriction of this equation to $U^{*}\left(L_{p}^{\infty}\right)$, we find that $y^{\prime} \Theta_{p}(u)=0$, where $y^{\prime}=\varepsilon(y), \varepsilon: U^{*}\left(S^{n}\right) \rightarrow U^{*}(*)$. Hence, if $m>1$, by the inductive hypothesis $y \cdot \Theta_{p}(u) \in R_{p}[[u]]$, which contradicts the minimality of $m$. But if $m=1$, then by considering the restriction of (I.4) to the group $U^{*}\left(S^{n}\right)$, we find that $-a=$ $\psi_{1}(0)+p y$, that is, $a \in R_{p}$.

\section{Appendix II ${ }^{1}$. The AdAms COnjecture}

The Adams conjecture is concerned with the computation of the image of the $J$-homomorphism in real $K$-theory (see [13]). Here is its precise formulation: for any bundle $\xi$ there is an integer $N$ such that $J\left(k^{N}\left(\Psi^{k}(\xi)-\xi\right)\right)=0$ for any $k \geq 1$. The Adams conjecture allows us to construct an upper bound for the image of the $J$-homomorphism. It was known that the Adams conjecture is valid for onedimensional and orientable two-dimensional bundles and also for their direct sums. To prove the Adams conjecture it is sufficient to verify it for the classifying bundles on Grassmann manifolds. We shall give an outline of Sullivan's proof of the Adams conjecture. $^{2}$ The basic idea is to transform the $K$-functor into another functor in which the Adams operations $\Psi^{k}$, so to speak, preserve the dimension of the "bundle". The precise meaning can be stated in the form of the following lemma.

Lemma II.1. Let $B_{n}$ be a sequence of complexes, $\gamma_{n}: E_{n} \rightarrow B_{n}$ sphere bundles with fibre $S^{n-1}, f_{n}: B_{n} \rightarrow B_{n-1}$ mappings such that $f^{*}\left(\gamma_{n+1}\right)=\gamma_{n} \oplus 1, f_{n} \sim \gamma_{n+1} \cdot h_{n}$, where $h_{n}: B_{n} \rightarrow E_{n+1}$ is a homotopy equivalence. Let $a_{n}: B_{n} \rightarrow B_{n}$ he a stable operation of the functor $\lim \left[, B_{n}\right]$, that is, $f_{n} a_{n} \sim a_{n+1} f$, which is invertible. If $J_{n}: B_{n} \rightarrow B G_{n}$ is the natural J-mapping, $G_{n} \approx\left(\Omega^{n-1} S^{n-1}\right)_{0}$, then $J_{n} \sim J_{n} a_{n}$.

Note that the operations $\Psi^{k}$ for the sequence $B_{n}=B O(n)$ do not satisfy the conditions of the lemma. Sullivan found an appropriate theory $K^{\wedge}(X)$, in which certain analogues of the $\Psi^{k}$ do satisfy the conditions of the lemma. Let $X$ be an arbitrary $C W$-complex. By the completion $\hat{X}$ of $X$ we mean the (unique) complex

\footnotetext{
${ }^{1}$ Appendix II was written hy Mishchenko.

${ }^{2}$ The proof of the Adaim conjecture began three years ago with Quillen's idea of applying a property of the étale-topology of Grassmann manifolds. (D. Quillen, Some remarks on étale homotopy theory and a conjecture of Adams. Topology 7 (1968), 111-116). In 1970, besides an account of Sullivan's proof, there appeared a proof by Quillen based on reducing the Adams conjecture to bundles with finite structure group and differing from his original idea.
} 
that satisfies the condition $[Y, \hat{X}]=\lim _{\{F, f\}}[Y, F]$. Here $\{F, f\}$ is the category of all mappings $f: X \rightarrow F$, where $F$ runs through the complexes having all their homotopy groups finite. We then put $K^{\wedge}(X)=\left[X, B O^{\wedge}\right]$. It is easy to see that the spaces $B O(n)^{\wedge}$ satisfy the conditions of the lemma if we take bundles with fibre $\left(S^{n-1}\right)^{\wedge}$ as the $\gamma_{n}$. On the other hand, since all the homotopy groups of $B G_{n}$ are finite, there exists a natural mapping $J^{\wedge}: B O(n) \rightarrow B G_{n}$ such that the diagram

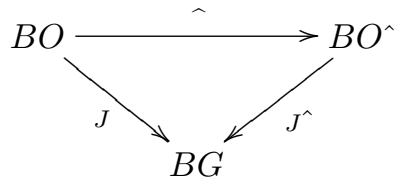

is commutative. Finally, we can define the operations $\left(\Psi^{k}\right)^{\wedge}$ in the groups $K^{\wedge}(X)$ such that $\left(\Psi^{k} x\right)^{\wedge}=\left(\Psi^{k}\right)^{\wedge}\left(x^{\wedge}\right)$. If we can prove that the operations $\left(\Psi^{k}\right)^{\wedge}$ preserve the geometric dimension of the bundle, in other words, that there exist mappings $\left(\Psi_{n}^{k}\right)^{\wedge}: B O(n)^{\wedge} \rightarrow B O(n)^{\wedge}$ such that $\left(\Psi^{k}\right)^{\wedge}=\lim \left(\Psi_{n}^{k}\right)^{\wedge}$, then the Adams conjecture follows from the lemma. To prove the latter assertion Sullivan uses the fact that the Grassmann manifolds $G_{n, k}$ are algebraic varieties over the field of rational numbers. Consequently the Galois group $\operatorname{Gal}(C, Q)$ acts on $G_{n, k}$. It turns out that the induced action on the étale cohomology with coefficients in a finite group is determined only by the representation of $\operatorname{Gal}(C, Q)$ in the group of permutations of all roots of unity, that is, the homomorphism $\operatorname{Gal}(C, Q) \rightarrow(\hat{Z})^{*}$. By using Artin's theorem on the isomorphism of étale cohomology with coefficients in a finite group and the usual cohomology of a manifold, we obtain an action of $(\hat{Z})^{*}$ on the space $\left(G_{n, k}\right)^{\wedge}$. An easy verification shows that the action of the element $(k) \in(\hat{Z})^{*}$ coincides with the operation $\left(\Psi^{k}\right)^{\wedge}$.

\section{REFERENCES}

[1] M. Atiyah and R. Bott, Notes on Lefschetz's fixed point theorem for elliptic complexes, Harvard University, Cambridge, Mass. 1964.

= Matematika 10:4 (1966), 101-139,

[2] V. M. Bukhshtaber, The Chern-Dold character in cobordisms. I. Mat. Sb. 83 (1970), 575-595. = Math. USSR-Sb. 12 (1970).

[3] V. M. Bukhshtaber, Spectral sequences connected with the Chern-Dold character, Uspekhi Mat. Nauk, to appear.

[4] V. M. Bukhshtaber and S. P. Novikov, Formal groups, power systems and Adams operators, Mat. Sb. 84 (1971), 116-153. = Math. USSR-Sb. 13 (1971).

[5] G. G. Kasparov, Invariants or classical lens spaces in the theory of cobordism, Izv. Akad. Nauk SSSR Ser. Mat. 33 (1969), 735-747. = Math. USSR-Izv. 3 (1969).

[6] P. E. Conner and E. E. Floyd, Differentiable periodic maps, Springer-Verlag, BerlinGöttingen-Heidelberg, 1964. Translation: Gladkie periodicheskie otobrazheniya, "Mir", Moscow 1969.

[7] D. Quillen, On the formal group laws of unoriented and complex cobordism theory, Bull. Amer. Math. Soc. 75 (1969), 1293-1298. MR 40\#6565.

[8] J. Milnor, Lectures on characteristic classes, Princeton University Lecture Notes, 1958. = Matematika 5:4 (1959).

[9] J. Milnor, On the cohordism ring $\Omega^{*}$ and a complex analogue. I. Amer. J. Math. 82 (1960), 505-521. MR 22\#9975. 
[10] A. S. Mishchenko, Manifolds acted on by the group $Z_{p}$ and fixed points, Mat. Zametki 4 (1968), 381-3S6.

= Math. Notes 4 (1968), 721-725.

[11] A. S. Mishchenko, Bordisms acted on by the group $Z_{p}$ and fixed points. Mat. Sb. 80 (1969), $307-313$.

= Math. USSR-Sb. 9 (1969), 291-296.

[12] S. P. Novikov, Homotopy properties of Thom complexes. Mat. Sb. 57 (1962), 407-442. MR $28 \# 615$.

[13] S. P. Novikov, New ideas in algebraic topology ( $K$-theory and its applications), Uspekhi Mat. Nauk 20:3 (1965), 41-66. MR 33\#716.

= Russian Math. Surveys 20:3 (1965), 37-62.

[14] S. P. Novikov, The methods of algebraic topology from the point of view of cobordism theory, Izv. Akad. Nauk SSSR Ser. Mat. 31 (1967), 885-951, MR 36\#4561. = Math. USSR-Izv. 1 (1967), 827-913.

[15] S. P. Novikov, The Adams operators and fixed points, Izv. Akad. Nauk SSSR Ser. Mat. 32 (1968), 1245-1264

= Math. USSR-Izv. 2 (1968), 1193-1211.

[16] R. Thom, Quelques propriétés globales des variétés differentiables, Comment. Math. Helv. 28 (1954), 17-86. MR 15-890.

Translation: Some properties in the large of differentiable manifolds, in the collection Rassloennye prostranstva i prilozheniya (Fibre spaces and applications), Izdat. Inost. Lit., Moscow 1958.

[17] T. Honda, Formal groups and zeta-functions, Osaka J. Math. 5 (1968), 199-213. MR $40 \# 2683$. = Matematika 13:6 (1969), 3-17.

[18] Chern Shiing-Shen, Complex manifolds, Textos de Matematica No. 5, Institute de Fisica e Matemàtica, Universidade do Recife, 1959. MR 22\#1920.

Translation: Kompleksnye mnogoobraziya, Izdat. Inost. Lit., Moscow 1961.

[19] T. tom Dieck, Steenrod-Operationen in Kobordism-Theorien, Math. Z. 107 (1968), 380-401, MR 39\#6302.

[20] D. Quillen, Elementary proofs of some results ofcobordism theory using Steenrod operations (preprint).

[21] V. M. Bukhshtaber, Two-valued formal groups. Some applications to cobordism, Uspekhi Mat. Nauk 26:3 (1971), 195-196. $=? ? ?$

[22] P. S. Landweber, Cobordism operations and Hopf algebras. Trans. Amer. Math. Soc. 129 (1967), 94-110. MR 36\#2145.

[23] J. F. Adams, Quillen's work on formal group laws and complex cobordism, Univ. of Chicago Lecture Notes Series, 1970. 